\title{
Coronavirus Disease 2019 Pneumonia and Acute Pancreatitis in a Young Girl
}

\author{
Paghunda Ehsan ${ }^{1}$, Muhammad Haseeb ${ }^{2}$, Zaraq Khan ${ }^{3}$, Aiman Rehan ${ }^{4}$, Romil Singh ${ }^{5}$
}

1. Medicine, Hayatabad Medical Complex Peshawar, Peshawar, PAK 2. Internal Medicine, Jinnah Hospital Lahore, Lahore, PAK 3. Internal Medicine, Hayatabad Medical Complex Peshawar, Peshawar, PAK 4. Internal Medicine, Dow University of Health Sciences, Karachi, PAK 5. Critical Care, Mayo Clinic, Rochester, USA

Corresponding author: Romil Singh, singh.romil20@gmail.com

\begin{abstract}
Severe acute respiratory syndrome coronavirus 2 pneumonia and acute pancreatitis are rarely reported in patients with coronavirus disease 2019 (COVID-19). We present the case of a 13-year-old girl who presented with nausea, vomiting, and abdominal pain for the last two days, along with a cough for the last week. She had a fever and tachycardia. Lung examination revealed reduced breath sounds, and abdominal examination showed tenderness in the epigastrium. COVID-19 polymerase chain reaction was positive, and her serum chemistry revealed elevated serum amylase and lipase. Abdominal computed tomography revealed diffuse inflammation of the pancreas with peripancreatic edema, and chest X-ray demonstrated diffuse infiltrates and pneumonic patches in both lungs. Her initial management included bowel rest, intravenous fluids, intravenous remdesivir, and azithromycin with supplemental oxygen based on the provisional diagnosis of COVID-19 pneumonia and acute pancreatitis. Her abdominal symptoms started improving, and dexamethasone was added to her regimen due to her worsened respiratory condition. She was symptom-free on day seven except for a mild cough. She was discharged on day eight with follow-up.
\end{abstract}

Review began 05/15/2021 Review ended 05/31/2021 Published 06/01/2021

๑) Copyright 2021

Ehsan et al. This is an open access article distributed under the terms of the Creative Commons Attribution License CC-BY 4.0., which permits unrestricted use, distribution, and reproduction in any medium, provided the original author and source are credited.
Categories: Internal Medicine, Infectious Disease

Keywords: covid-19, sars-cov-2, pneumonia, acute pancreatitis, coronavirus disease 2019

\section{Introduction}

Coronavirus disease 2019 (COVID-19) has spread rapidly around the world, causing a pandemic and affecting individuals of all ages [1,2]. A study reported that around $4 \%$ of COVID-19 patients were below the age of 18 in the United States [3]. COVID-19 has a typical manifestation of the respiratory system and generally presents with fever, cough, dyspnea, and fatigue. COVID-19 has also demonstrated signs and symptoms in the gastrointestinal (GI) system, cardiovascular system, and neurological system [4-7]. Nausea, vomiting, abdominal pain, and diarrhea have also been reported as an initial manifestation of severe acute respiratory syndrome coronavirus 2 (SARS-CoV-2) [8]. Acute pancreatitis induced by COVID-19 pneumonia in children is rarely reported in the literature [9]. Herein, we describe a case of acute pancreatitis associated with COVID-19 pneumonia in a young girl.

\section{Case Presentation}

A 13-year-old girl without a previous medical problem was brought to the emergency department for nausea, persistent nonbloody vomiting, and abdominal pain for the last two days, along with one episode of loose stool. Abdominal pain was sudden in onset, progressive, and located in the epigastrium. She also had a mild cough and fever for the last week. She was previously healthy with no history of fever and ill contacts. Initial evaluation revealed a temperature of $100^{\circ} \mathrm{F}$, tachypnea (31/minute), tachycardia (103/minute), and borderline oxygen saturation (93\%). On clinical examination, she looked ill, and her lungs were clear with reduced breath sounds in the lower lobe of the right lung. Abdominal examination was unremarkable except for tenderness in the epigastrium. A nasal swab for COVID-19 polymerase chain reaction was done as part of the regular screening which was positive. Initial laboratory investigations revealed elevated serum lipase, amylase, erythrocyte sedimentation rate (ESR), and C-reactive protein (CRP) (Table 1). 


\section{Cureus}

\begin{tabular}{|c|c|c|c|}
\hline Parameter & Day 1 & Day 6 & Reference range \\
\hline White blood cells & 10,007 & 8,899 & $4,000-11,000 / \mathrm{mm}^{3}$ \\
\hline Red blood cells & 5.1 & 5.0 & $4.35-5.65$ million cells $/ \mathrm{mm}^{3}$ \\
\hline Hemoglobin & 12 & 12 & $12-15.5 \mathrm{mg} / \mathrm{dL}$ \\
\hline Aspartate aminotransferase & 46 & 37 & 8-35 IU/L \\
\hline Alanine aminotransferase & 44 & 41 & 7-35 IU/L \\
\hline Serum lipase & 2,331 & 877 & $0-160 \mathrm{IU} / \mathrm{L}$ \\
\hline Serum amylase & 598 & 189 & 30-110 IU/L \\
\hline Serum triglyceride & 135 & 134 & $<150 \mathrm{mg} / \mathrm{dL}$ \\
\hline Serum creatinine & 1.0 & 0.9 & $0.7-1.2 \mathrm{mg} / \mathrm{dL}$ \\
\hline Blood urea nitrogen & 19 & 20 & $8-20 \mathrm{mg} / \mathrm{dL}$ \\
\hline Lactate dehydrogenase & 419 & 295 & 140-280 IU/L \\
\hline Erythrocyte sedimentation rate & 37 & 23 & $<22$ \\
\hline C-reactive protein & 14 & 04 & $<0.2 \mathrm{mg} / \mathrm{dL}$ \\
\hline Total bilirubin & 1.5 & 1.3 & $0.3-1.2 \mathrm{mg} / \mathrm{dL}$ \\
\hline D-dimer & 1,431 & 487 & $<250 \mathrm{ng} / \mathrm{L}$ \\
\hline Creatıne kınase & 202 & 139 & $30-135$ IU/L \\
\hline
\end{tabular}

TABLE 1: Initial blood workup.

Abdominal ultrasonography was nonsignificant with no evidence of gallstone, and the pancreas was not visualized. Abdominal computed tomography (CT) was performed, which revealed diffuse inflammation of the pancreas with peripancreatic edema without necrosis, consistent with acute pancreatitis (Figure 1). Her chest X-ray demonstrated diffuse infiltrates and pneumonic patches in the lower lobe of both lungs (Figure 2). 


\section{Cureus}

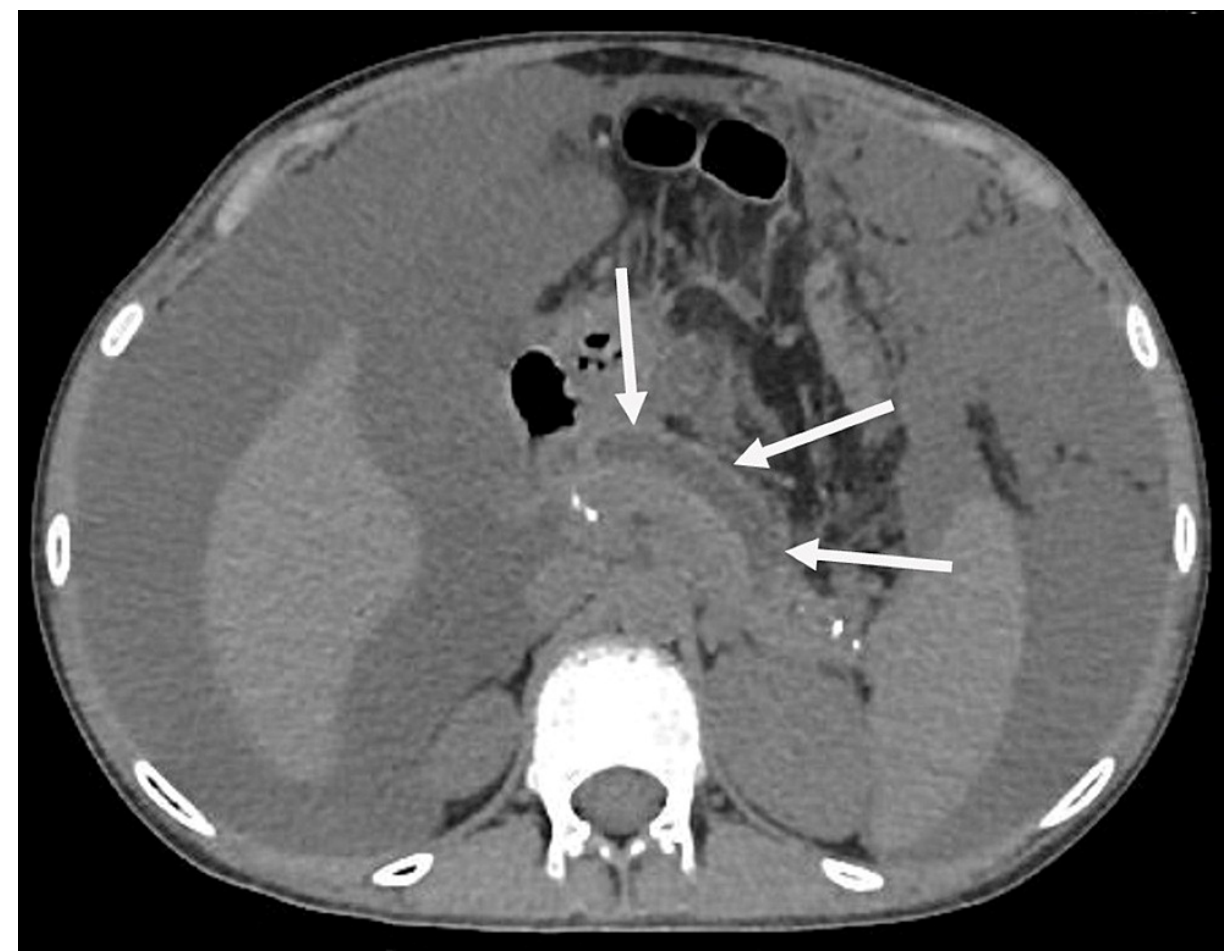

FIGURE 1: CT abdomen showing pancreatic inflammation and edema (white arrows).

CT: computed tomography 


\section{Cureus}

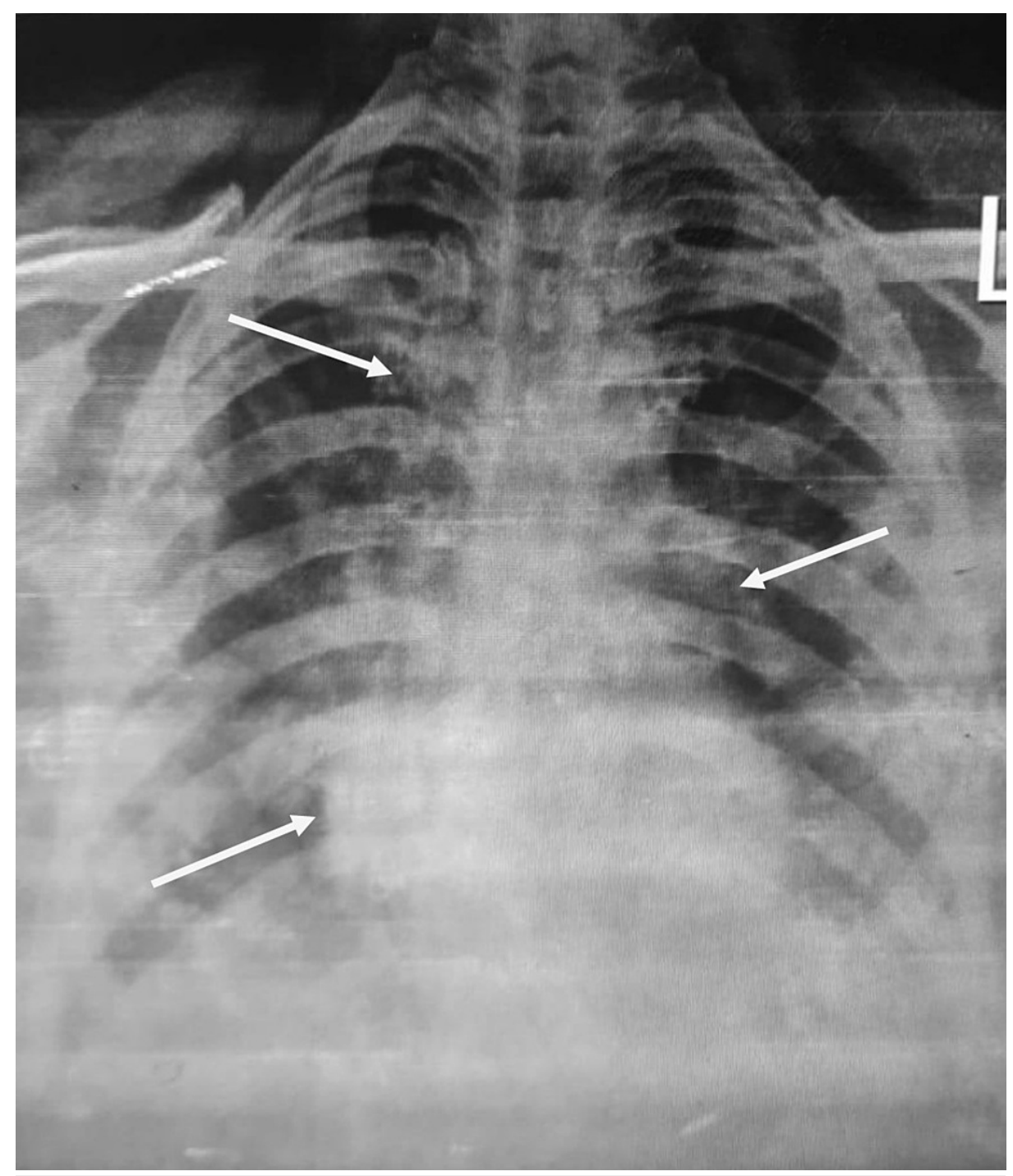

\section{FIGURE 2: Chest X-ray showing infiltrates and pneumonic patches in both lungs.}

Diagnostic workup for infection, including blood culture and sputum culture, were negative. She had no history of previous pancreatitis and no recognized risk factors such as family history, trauma, gallstone, alcohol consumption, or medication exposure. Due to the lack of any identifiable risk factor, she was diagnosed with acute pancreatitis and pneumonia due to COVID-19. She was admitted to the gastroenterology ward to treat acute pancreatitis, and infectious disease consultation was taken to manage COVID-19 pneumonia.

Her initial management included bowel rest, dietary restrictions, intravenous fluids, antiemetics, and analgesics. For COVID-19 pneumonia, she was commenced on intravenous remdesivir $(5 \mathrm{mg} / \mathrm{kg}$ ) and azithromycin $(20 \mathrm{mg} / \mathrm{kg})$ with supplemental oxygen. Her abdominal symptoms started improving on day two; however, her respiratory system worsened with a gradual drop in oxygen saturation to $85 \%$. She was shifted to the intensive care unit for aggressive management. She was commenced on dexamethasone $(0.15$ $\mathrm{mg} / \mathrm{kg}$ ) in addition to azithromycin and remdesivir, and she required oxygen at $3 \mathrm{~L} /$ minute by nasal cannula. She was stable on day four of hospitalization and was symptom-free on day six except for a mild cough. She received seven days of treatment and was discharged on day eight with follow-up. She was doing well on her recent visit seven days later. Her laboratory investigations on day one and day six are shown in Table 1.

\section{Discussion}

Acute pancreatitis is a medical emergency requiring immediate diagnosis and resuscitation, with a mortality rate up to 30\% in severe cases. Multiple conditions can induce acute pancreatitis. Alcohol consumption, gall stone, and hypertriglyceridemia are among the common causes of acute pancreatitis [10]. Many viruses have also been implicated as causative agents of acute pancreatitis. However, COVID-19-induced pancreatitis is not reported widely in the literature, and only a small number of cases have been reported. A study from 
China underlined that $17 \%$ of COVID-19 cases had elevated pancreatic enzymes, and a case series of three COVID-19 patients described acute pancreatitis in two cases [11,12]. Kataria et al. reported a case of acute pancreatitis as an initial manifestation of COVID-19 [9]. Our case involved a 13-year-old girl who presented with vomiting, abdominal pain, dry cough, and fever, which was diagnosed as acute pancreatitis and COVID19 pneumonia.

The mechanism of acute pancreatitis due to COVID-19 is not yet defined. COVID-19 is an inflammatory disease characterized by a widespread immune response throughout the body. Angiotensin-converting enzyme 2 (ACE2) receptors are responsible for attachment and invasion inside the human cells [13,14]. These receptors are predominately expressed in alveolar cells of the lungs, epithelial lining of the GI tract, and pancreatic islets, responsible for GI manifestation and elevation of pancreatic enzymes in COVID-19 patients, along with respiratory manifestations [15]. Possible mechanisms have been explained for COVID19-induced pancreatitis. SARS-CoV-2 may directly cause acute pancreatitis due to the higher expression of ACE2 receptors in pancreatic islets. Acute pancreatitis can be secondary to intense inflammatory response and cytokine storm induced by COVID-19 infection [16,17]. In our case, the patient had laboratory evidence of marked systemic inflammation with increased ESR, CRP, and D-dimer.

Diagnosis of acute pancreatitis is by clinical, serology, and imaging modalities. Serum amylase and lipase are highly specific, and three times greater than the standard value is considered diagnostic. Ultrasound and CT of the abdomen help in diagnosis and ruling any other complication of acute pancreatitis. Management of acute pancreatitis is conservative and symptomatic [10]. Treatment involves bowel rest, resuscitation with isotonic fluids, and analgesics. The standard of care in children with acute pancreatitis includes painkillers and crystalloid intravenous fluid [18]. Recently published guidelines for critically ill patients with SARS$\mathrm{CoV}-2$ recommend the conservative fluid strategy and maintaining fluid balance [19].

\section{Conclusions}

Our case highlights the need for an immediate evaluation of COVID-19 patients who present with GI manifestations for the possibility of acute pancreatitis. COVID-19 has a variable presentation due to the involvement of other systems and leads to poor prognosis and prolonged hospital stay if the case is not managed appropriately. Our case encourages physicians to investigate the GI manifestations carefully in patients with SARS-CoV-2 to prevent delay in management and potential complications. There is also a need to identify a possible relationship between acute pancreatitis and COVID-19.

\section{Additional Information \\ Disclosures}

Human subjects: Consent was obtained or waived by all participants in this study. Conflicts of interest: In compliance with the ICMJE uniform disclosure form, all authors declare the following: Payment/services info: All authors have declared that no financial support was received from any organization for the submitted work. Financial relationships: All authors have declared that they have no financial relationships at present or within the previous three years with any organizations that might have an interest in the submitted work. Other relationships: All authors have declared that there are no other relationships or activities that could appear to have influenced the submitted work.

\section{References}

1. Wu Z, McGoogan JM: Characteristics of and important lessons from the coronavirus disease 2019 (COVID19) outbreak in China: summary of a report of 72314 cases from the Chinese Center for Disease Control and Prevention. JAMA. 2020, 323:1239-42. 10.1001/jama.2020.2648

2. Khan I, Sarwar A, Ahmed Z: Atypical case of COVID-19 associated Kawasaki disease in an eight-year-old Pakistani boy. Cureus. 2020, 12:e10670. 10.7759/cureus.10670

3. CDC COVID-19 Response Team: Coronavirus disease 2019 in children - United States, February 12-April 2, 2020. MMWR Morb Mortal Wkly Rep. 2020, 69:422-6. 10.15585/mmwr.mm6914e4

4. Singh R, Kashyap R, Hutton A, Sharma M, Surani S: A review of cardiac complications in coronavirus disease 2019. Cureus. 2020, 12 :e8034. 10.7759/cureus.8034

5. Menon T, Gandhi SAQ, Tariq W, et al.: Impact of chronic kidney disease on severity and mortality in COVID19 patients: a systematic review and meta-analysis. Cureus. 2021, 13:e14279. 10.7759/cureus.14279

6. Menon T, Sharma R, Kataria S, et al.: The association of acute kidney injury with disease severity and mortality in COVID-19: a systematic review and meta-analysis. Cureus. 2021, 13:e13894. 10.7759/cureus.13894

7. Singh R, Shiza ST, Saadat R, Dawe M, Rehman U: Association of Guillain-Barre syndrome with COVID-19: a case report and literature review. Cureus. 2021, 13:e13828. 10.7759/cureus.13828

8. Menon T, Sharma R, Earthineni G, et al.: Association of gastrointestinal system with severity and mortality of COVID-19: a systematic review and meta-analysis. Cureus. 2021, 13:e13317. 10.7759/cureus.13317

9. Kataria S, Sharif A, Ur Rehman A, Ahmed Z, Hanan A: COVID-19 induced acute pancreatitis: a case report and literature review. Cureus. 2020, 12:e9169. 10.7759/cureus.9169

10. Gapp J, Chandra S: Acute pancreatitis. StatPearls [Internet]. StatPearls Publishing, Treasure Island, FL; 2020.

11. Wang F, Wang H, Fan J, Zhang Y, Wang H, Zhao Q: Pancreatic injury patterns in patients with coronavirus 


\section{Cureus}

disease 19 pneumonia. Gastroenterology. 2020, 159:367-70. 10.1053/j.gastro.2020.03.055

12. Hadi A, Werge M, Kristiansen KT, Pedersen UG, Karstensen JG, Novovic S, Gluud LL: Coronavirus disease-19 (COVID-19) associated with severe acute pancreatitis: case report on three family members. Pancreatology. 2020, 20:665-7.10.1016/j.pan.2020.04.021

13. Jin X, Lian JS, Hu JH, et al.: Epidemiological, clinical and virological characteristics of 74 cases of coronavirus-infected disease 2019 (COVID-19) with gastrointestinal symptoms. Gut. 2020, 69:1002-9. 10.1136/gutjnl-2020-320926

14. Neupane K, Ahmed Z, Pervez H, Ashraf R, Majeed A: Potential treatment options for COVID-19: a comprehensive review of global pharmacological development efforts. Cureus. 2020, 12:e8845. 10.7759/cureus.8845

15. Su S, Wong G, Shi W, et al.: Epidemiology, genetic recombination, and pathogenesis of coronaviruses . Trends Microbiol. 2016, 24:490-502. 10.1016/j.tim.2016.03.003

16. Liu F, Long X, Zhang B, Zhang W, Chen X, Zhang Z: ACE2 expression in pancreas may cause pancreatic damage after SARS-CoV-2 infection. Clin Gastroenterol Hepatol. 2020, 18:2128-30. 10.1016/j.cgh.2020.04.040

17. Khan I, Ahmed Z, Sarwar A, Jamil A, Anwer F: The potential vaccine component for COVID-19: a comprehensive review of global vaccine development efforts. Cureus. 2020, 12:e8871. 10.7759/cureus.8871

18. Tenner S, Baillie J, DeWitt J, Vege SS: American College of Gastroenterology guideline: management of acute pancreatitis. Am J Gastroenterol. 2013, 108:1400-15. 10.1038/ajg.2013.218

19. Alhazzani W, Møller MH, Arabi YM, et al.: Surviving sepsis campaign: guidelines on the management of critically ill adults with coronavirus disease 2019 (COVID-19). Crit Care Med. 2020, 48:e440-69. 10.1097/CCM.0000000000004363 